\title{
The Diagnostic History of a New Electromagnetic Powder Deposition System
}

By:

J.L. Bacon

D.G. Davis

R.J. Pollizzi

R.L. Sledge

J.R. Uglum

R.C. Zowarka

United Thermal Spray Conference, Sept. 15-18, 1997, Indianapolis Indiana

ASM International

PR -230

Center for Electromechanics

The University of Texas at Austin

PRC, Mail Code R7000

Austin, TX 78712

(512) $471-4496$ 


\title{
The Diagonostic History of a New Electromagnetic Powder Deposition System
}

\author{
J.L. Bacon, D.G. Davis, R.J. Polizzi, R.L. Sledge, J.R. Uglum, and R.C. Zowarka \\ Center for Electromechanics \\ The University of Texas at Austin \\ Austin, Texas
}

\begin{abstract}
This paper describes the diagnostic tools used in the development of a new electromagnetic powder deposition system. The instrumentation, interpretation of data, and subsequent decisions regarding the direction of system development are discussed. Important system parameters, their impact on system performance, and techniques to measure them are presented.

The electromagnetic powder deposition system is based on railgun technology developed by the Department of Defense. The system drives an ionized plasma sheet down the length of a railgun, reaching a final plasma velocity of $4 \mathrm{~km} / \mathrm{sec}$. The high velocity plasma, in turn, snowplows [1] a shock compressed gas column in front of it. This gas column sweeps through a powder cloud and accelerates it by viscous drag to a final velocity of $2 \mathrm{~km} / \mathrm{sec}$.

Important system parameters include particle velocity, gas velocity, gas column pressure, and plasma propagation and velocity. Diagnostic tools include pressure transducers, a high speed digital framing camera, fiber optics and magnetic probes.
\end{abstract}

\section{AN ELECTROMAGNETIC POWDER DEPOSITION sys-} tem utilizes electrical energy to drive an argon gas column through a powder cloud and accelerate the powder to velocities of up to $2 \mathrm{~km} / \mathrm{sec}$. A metal powder particle traveling at this speed has sufficient kinetic energy that, upon impact with a substrate, it and an equal mass of substrate will melt.
The gas column is accelerated using a 'railgun' powered by a capacitor bank. The bank is used to produce a very short duration, high magnitude electrical pulse (a peak current magnitude of $160 \mathrm{kA}$ and 120 microseconds duration) which is conducted by a segment of argon plasma called the armature. As current increases, energy is deposited in the argon plasma increasing its temperature. Current moving through the plasma armature generates an electromagnetic Lorentz force which acts on the plasma, accelerating it down the length of the railgun (figure 1). As the armature moves down the railgun, it snowplows a column of gas in front of it. The gas column is shock compressed so that its density and length continues to increase with time. Powder is accelerated by viscous drag forces generated by the gas column.

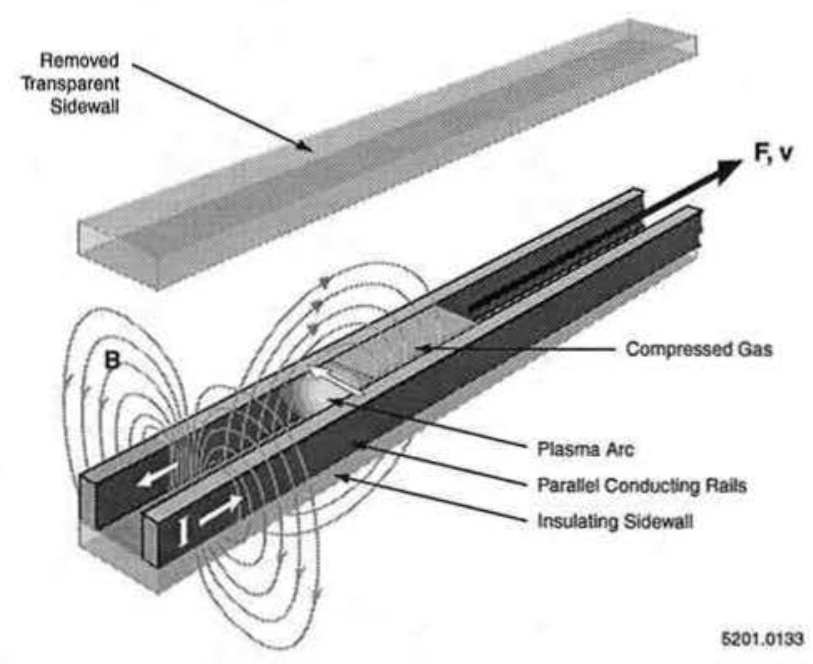

Figure 1. The plasma armature is accelerated down the length of the railgun by an electromagnetic Lorentz force generated by the by the interaction of the magnetic fields surrounding the rails and the current flowing through the armature. 


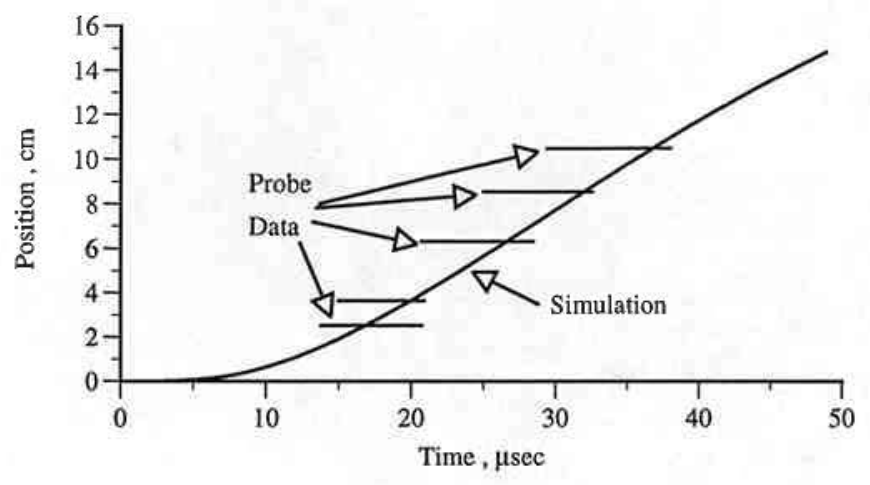

Figure 2. A comparison between predicted and measured armature position. The horizontal bars at each probe location represent the total time extent of signal activity over 5 different shots. In all cases the expected time for the actual armature arc is at the right of the range. It can be seen that this is generally where the predicted curve falls.

The ability to snowplow a column of gas is essential to the EPD process. A bench test railgun was fabricated and tested to determine whether the snowplow action could be experimentally produced. The railgun's performance was simulated [2] to predict the plasma armature's position through time, the shock gas column pressure and the veloci- ty of test beads accelerated in the railgun. The same quantities were measured during the testing and compared to the simulated results. Figure 2 shows the predicted position of the armature through time and the measured positions using B-dot probes [3,4] (magnetic field probes) and fiber optics. Simulation results were based on the assumption of snowplow. If snowplow had not occurred the plasma armature would accelerate to a velocity far greater than predicted. The excellent correlation between predicted and measured positions indicates that the snowplow action was successfully achieved.

\section{Diagnostic Tool Description}

The location and movement of the plasma armature was measured in two ways. The first was to detect its magnetic field. A magnetic flux encircles the current flowing in the plasma armature. The flux is shown pictorially in figure 3. This flux may be sensed by a small coil of wire placed external to the bore, parallel to the axis of the gun. Faraday's law indicates that the voltage induced in the coil of wire is proportional to the time derivative of the flux passing through the center of the coil. Because the voltage is proportional to the time derivative of the magnetic field, $\mathrm{B}$, these probes are commonly referred to as B-dot probes. The axial B-dot graph in figure 3a shows the magnitude of the magnetic flux that links the small coil as the distribution of flux associated

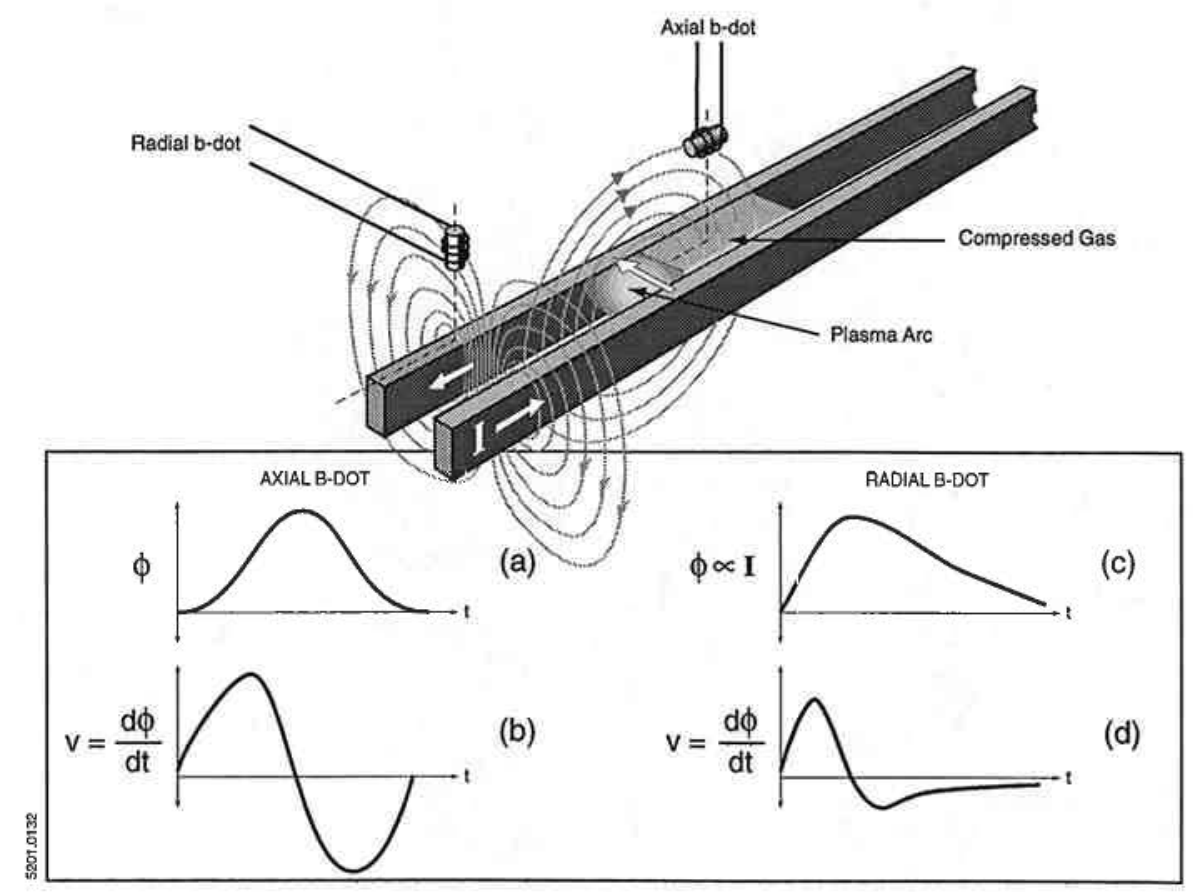

Figure 3. Two types of magnetic probes called B-dot probes used to sense the passing of the plasma armature. 
with the armature approaches and then recedes from the coil. The direct voltage output of the coil, the derivative of the flux, is shown in the figure $3 \mathrm{~b}$. When several of these coils are placed at discrete locations along the bore the displacement time history of the plasma arc may be mapped. A good indication of the center of the current distribution of the arc is where the signal from axial B-dot probe crosses zero. Further the displacement time history may be fit with a curve and extracting the derivative of that curve provides the velocity time history of the arc in bore.

Another type of B-dot probe is often used in the diagnostics of railguns. This probe is referred to as a radial B-dot. Once again, this probe is external to the bore, but the coil of wire forming the probe points radially away from the bore of the gun. The current flowing in the rails of the accelerator forms a second pattern of flux that is filling the bore of the gun as the armature progresses down bore. This second distribution of flux is also pictured in figure 3. In this case Ampere's law defines the flux as directly proportional to the current supplying the gun, shown in figure $3 \mathrm{c}$. The derivative of the flux is shown in figure $3 \mathrm{~d}$. In this case the direct output of the coil, figure $3 \mathrm{~d}$, may be archived on a digital oscilloscope and then integrated to show the amount of current that has passed any given probe. This data may be used in two ways: 1) similar to the axial B-dot, it can indicate time of arrival of the armature and 2) it indicates the amount of current that has passed a given location of the gun. The second feature of this probe allows diagnosis of malfunction of the accelerator such as two arcs running simultaneously in the gun.

The two types of magnetic probes are powerful tools in mapping performance of electric guns, and we have used them in our research to confirm snowplow action in the powder gun accelerator.

The second diagnostic tool used to detect the plasma armature was fiber optics. The conducting rails of the railgun are physically separated from each other with electrical insulating members referred to as "sidewalls". One of these sidewalls was made of polycarbonate, which is transparent. Fiber optic probes were mounted to the side of this polycarbonate sidewall so that they could detect light emitted by the passing plasma sheet. Figure 4 shows the fiber optic placement on the railgun and the plasma armature's passage down the railgun. The fiber optic probes were connected to light sensing diodes, which produce a voltage proportional to the intensity of the light picked up by the fiber optic probe.

As mentioned earlier, the plasma sheet snowplows a column of gas down the length of the railgun. The gas column

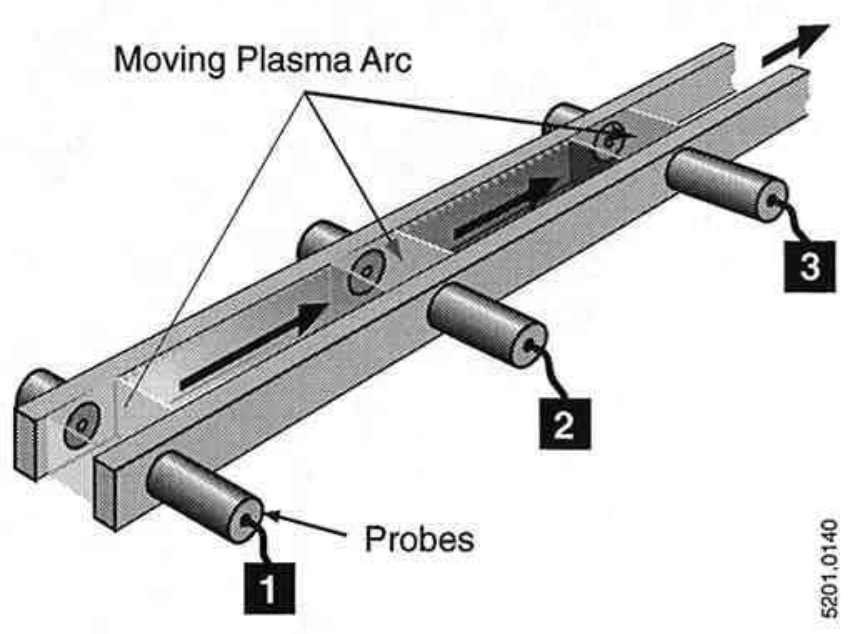

Figure 4. The placement of the fiber optic probes and the movement of the plasma armature down the length of the railgun past each of the fiber optic probes. Fiber optic 1 should detect the passage of the plasma armature first, followed by fiber optic 2 and then fiber optic 3 .

is accelerated to a velocity in excess of the speed of sound, creating a shock front. One of the sidewalls had two holes drilled through it and pressure transducers were mounted on the sidewall at the hole locations so that they could detect the pressure variations within the shock compressed gas.

To this point, diagnostic tools have been discussed which provide data on the plasma sheet and the shocked gas column. The final evidence of the viability of Electromagnetic Powder Deposition System was that the shocked gas column accelerated particles to predicted velocity. 330 micron diameter glass beads were placed on a fiber optic strand inserted into the railgun through the second sidewall. Photographs of the beads in motion were taken with an Imacon 468 high speed digital framing camera obtained from Hadland Photonics. The camera is capable of taking eight exposures with a minimum of 10 nanosecond exposure time and 20 nanosecond inter-frame separation. Photographs were taken at 1 microsecond frame separation and particle velocities were calculated using a software package provided with the Hadland camera.

During the course of testing, several problems associated with the diagnostic tools were encountered and solved. The B-dot probes and pressure transducers had to be isolated from the strong magnetic and electric fields. The fiber optic probes had to be filtered so they detected only the light emitted by the plasma sheet instead of the full spectrum of light in the railgun. Problems encountered, and remedial steps taken to successfully collect data, are discussed in the next section. 


\section{Diagnostic Tool Development}

One of the diagnostic tools used to detect the plasma armature's position was axial B-dot probes. Two probes were initially mounted on the sidewall of the railgun directly above the electrical buswork connection. This placement exposed the probes to the electromagnetic fields generated by the buswork. Figure 5 shows the voltage signal generated with a probe in this position. The signal showed an initial noise spike at the time current was initiated.

The B-dot probes were then moved to the other sidewall, away from the electrical buswork. Figure 6 shows an axial B-dot probe signature for this location, along with system current for reference. The initial noise spike is missing, and a larger negative voltage is observed. The zero crossing of the B-dot signal just after peak system current corresponds to the center of the armature passing the probe.

Fiber optics were used in addition to the B-dot probes to determine the position of the plasma armature. Initially, four fiber optic strands were mounted on the polycarbonate sidewall spaced at $4 \mathrm{~cm}$ down the length of the railgun. When the plasma armature was accelerated from the breech of the railgun to the muzzle, the first fiber optic channel should show a signal followed sequentially by the second, third, and finally the fourth signal. This did not occur in the initial tests when the full light spectrum was viewed. Figure 7 shows waveforms typical of these early tests, in which no distinct time pattern of events was found. Note that the signals arrive in the wrong order of $1,3,2$, then 4 and trail off in the order of $1,2,4$, and 3 .

This problem was solved when photographs from the Hadland camera revealed a thin black line moving up the gun, crossing the B-dot probe location at the same time as its zero crossing. This thin black line was obviously the plasma armature, and it was at a sufficient temperature to be radiating in the ultra-violet region of the spectrum. Viewing the ultra-violet (UV) light was possible using a band-pass filter, quartz fibers, and a polycarbonate front sidewall. The filter allowed wavelengths between $250 \mathrm{~nm}$ to $390 \mathrm{~nm}$ to pass with a peak efficiency of $74 \%$ at $325 \mathrm{~nm}$. The materials used were important because standard glass does not efficiently pass UV light. The UV method produced repeatable data with the expected progression from breech to muzzle (figure 8).

In debugging the greatest problems were experienced with the pressure transducers, an important tool for verifying the snowplow mechanism. At first, the pressure transducer traces exhibited a great deal of noise (figure 9). Since the transducers where directly exposed to the plasma, it was pos-

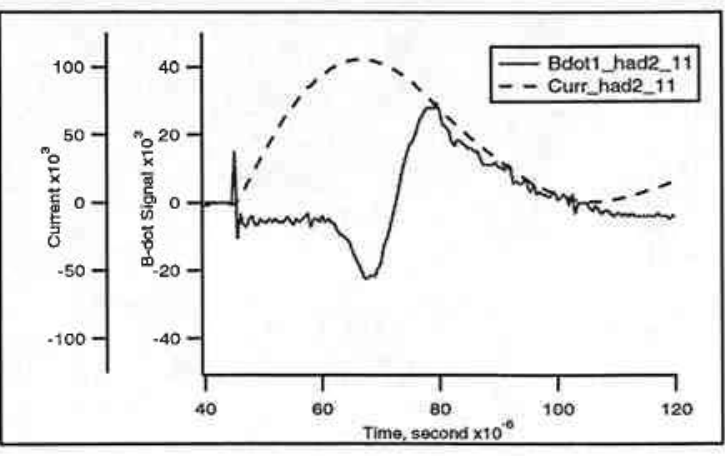

Figure 5. B-dot signal when probe was located on the buswork side of the railgun.

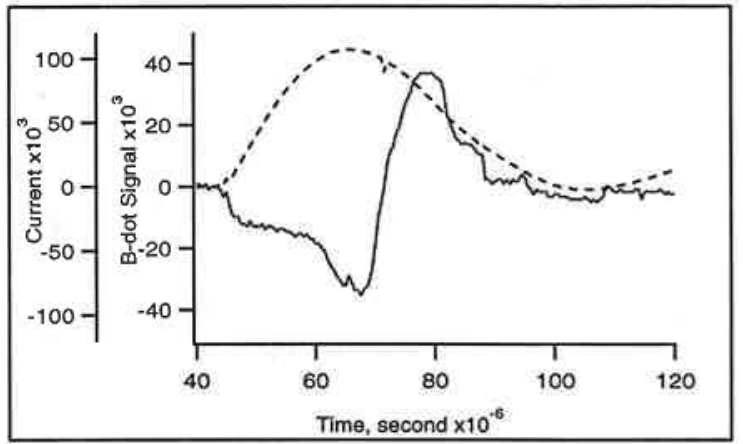

Figure 6. B-dot signal provides accurate passing time of the armature for purposes of position and velocity measurement.

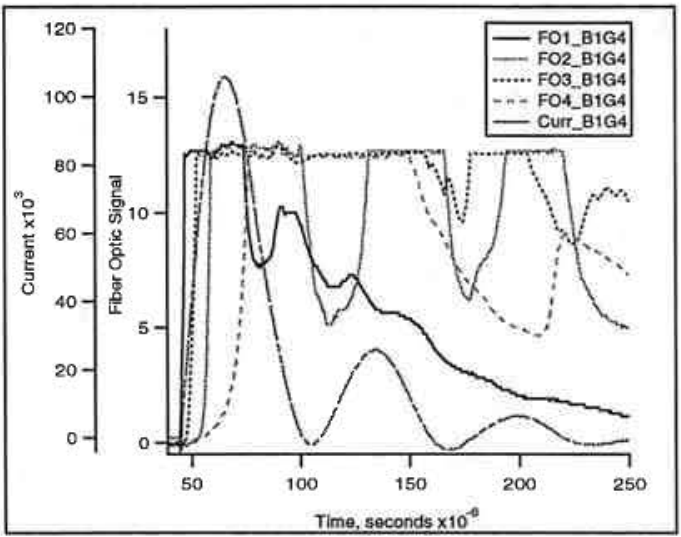

Figure 7. Fiber optic data using no filtering.

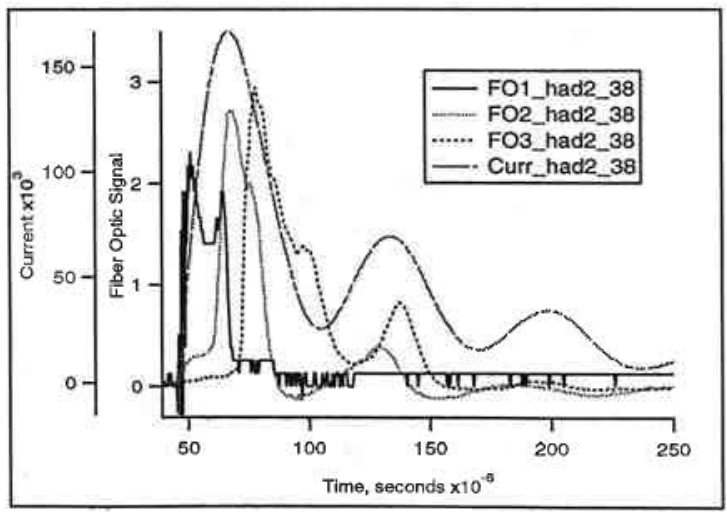

Figure 8. Fiber optic data using a UV band-pass filter. 


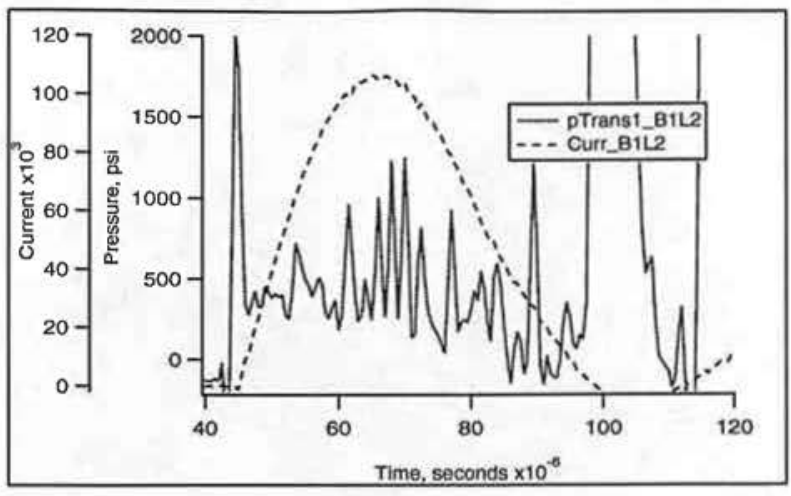

Figure 9. Pressure transducer signature from test with pressure transducer exposed to plasma.

sible that the transducer diaphragm was either shorting out to ground or picking up an electrical charge from the plasma. Kapton tape was placed over the face of the transducers to prohibit plasma charge coupling or at least delay coupling until after the acceleration event. This benefit is shown in figure 10. Most of the jagged nature of the waveform had been eliminated. There is still an apparent initial pressure rise which is probably induced by the current pulse but it has a much lower magnitude than the large spike seen previously. The shock front arrival is clearly seen as a sharp rise to 1,000 psi.

Two tests were performed to determine the cause of the initial pressure increase. The pressure transducer hole was plugged with a set screw and the transducer was mounted just behind it such that no bore pressure was measured. The test was then repeated. Figure 11 shows that the initial spike was due to electromagnetic (EM) noise caused by the high $\mathrm{di} / \mathrm{dt}$ present at current initiation. Next, a grounded copper shield was placed around the pressure transducer lead (figure 12). Note the reduction in peak noise from 708 to $415 \mathrm{psi}$.

\section{Experimental Verification of Snow Plow}

Once all of the diagnostics were debugged it was possible to determine if the snow plow process was working as predicted. A sequence of tests were performed which used all of the diagnostic tools available to determine:

1) if the plasma armature was formed

2) if a gas column was snowplowed in front of the plasma armature

3) the pressure of the snowplowed column of argon gas

4) the velocity of gas beads accelerated by the column of argon gas

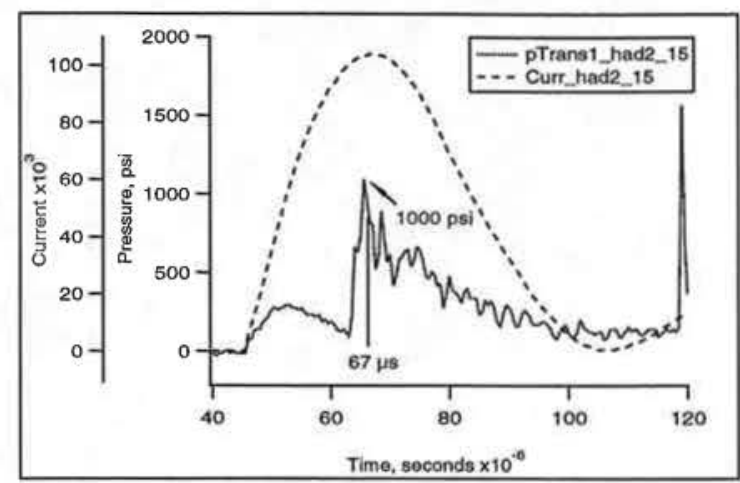

Figure 10. Pressure transducer signature from test with Kapton tape over pressure transducer.

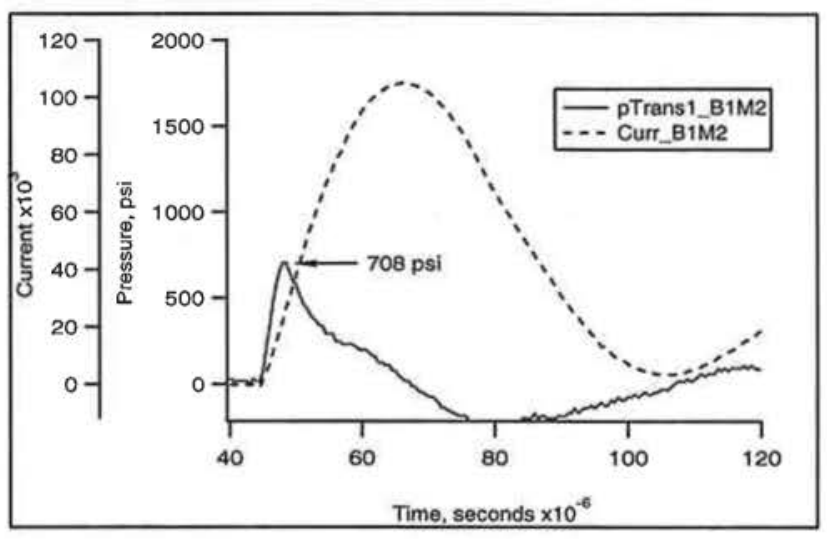

Figure 11. Test to determine effect of EM noise on pressure transducer positioned next to plugged port.

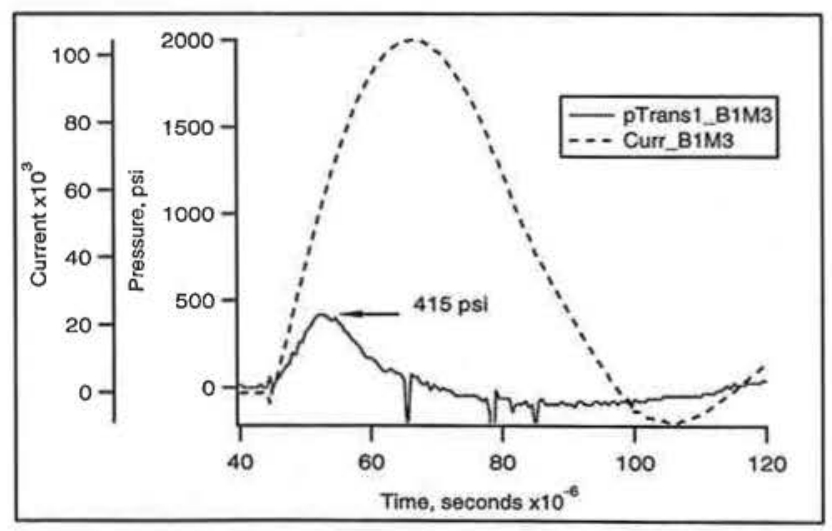

Figure 12. Test to determine effect of EM noise on pressure transducer positioned next to plugged port, with electrical shielding around the transducer cable. 
All of the tests were carried out with a $400 \mu \mathrm{F}$ capacitor bank charged to $8 \mathrm{kV}$. The diagnostics used to characterize the performance of the electromagnetic powder deposition system consisted of :

1) the Hadland camera to photograph the glass beads

2) a pressure transducer located at a distance of $2.5 \mathrm{~cm}$ from the plasma armature initiation point

3) two axial B-dot probes, one at $3.5 \mathrm{~cm}$ and the other at $8.5 \mathrm{~cm}$ from the plasma armature initiation point

4) and three fiber optic probes located at 2.5, 6.5, and 10.5 $\mathrm{cm}$ from the plasma armature initiation point.

Figure 13 shows the location of the diagnostics on the lower half of the railgun. Many tests were performed with this configuration. Here we will consider only a sub-set of 5 shots, specifically numbers $36,37,38,40$, and 44 , because good photographs were obtained on these shots of the glass beads in motion.

To provide a statistical feeling for the reproducibility of the diagnostics used to measure the motion of the arc armature, figures 14 through 18 show multi-shot waveform overlays for each of the B-dot and fiber optic probes used.

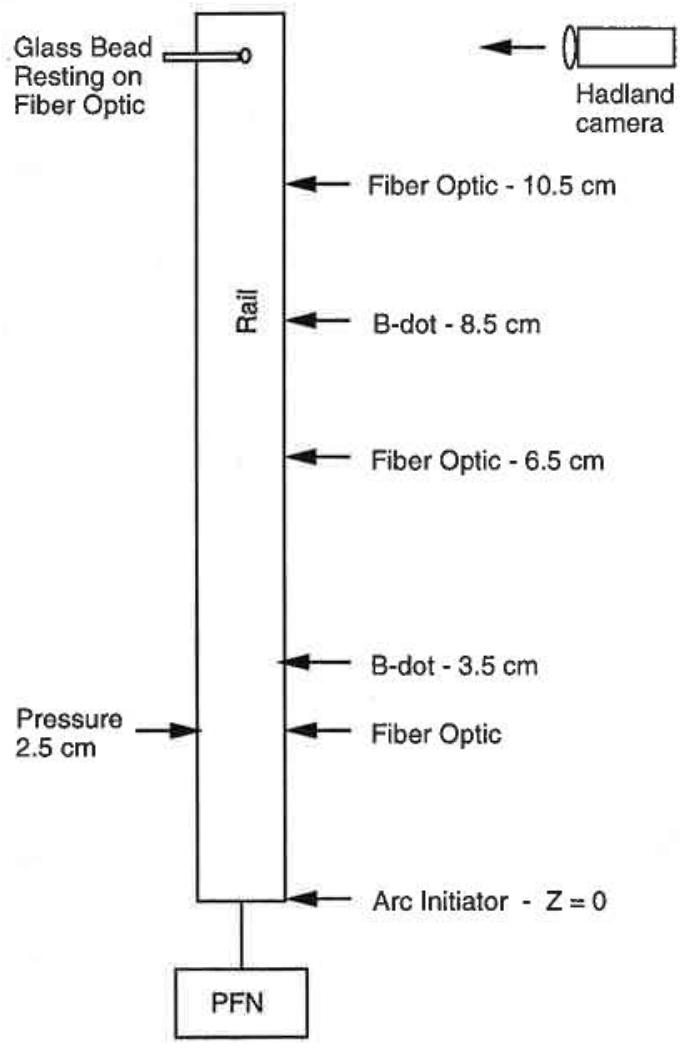

Figure 13. Diagnostic locations on the lower half of the railgun.

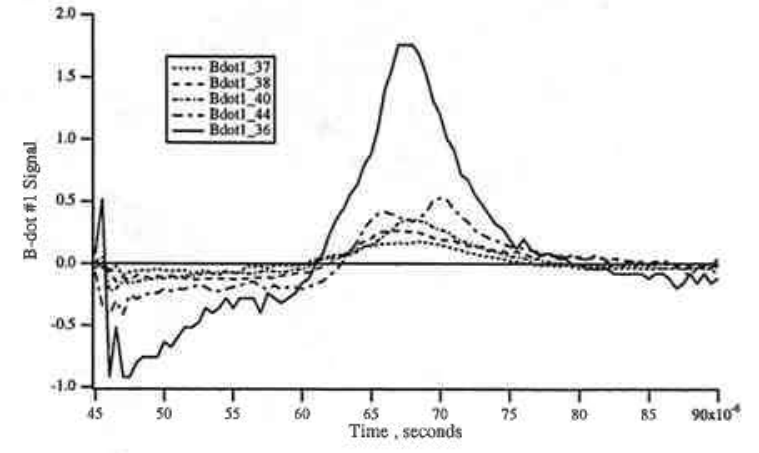

Figure 14. Overlay of B-dot \#1 waveforms.

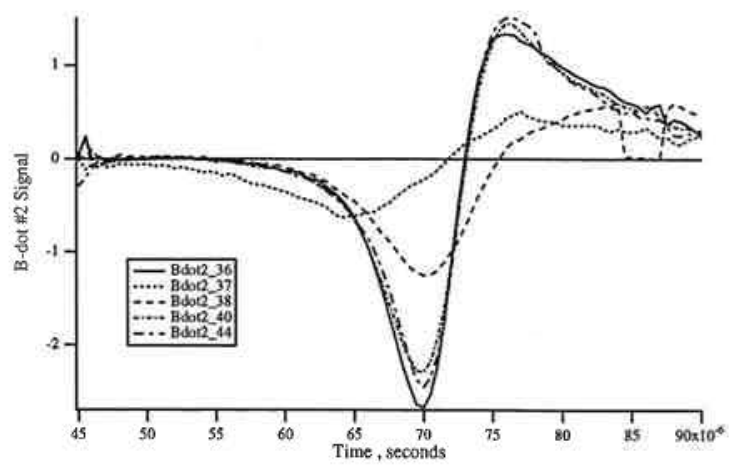

Figure 15. Overlay of B-dot \#2 waveforms.

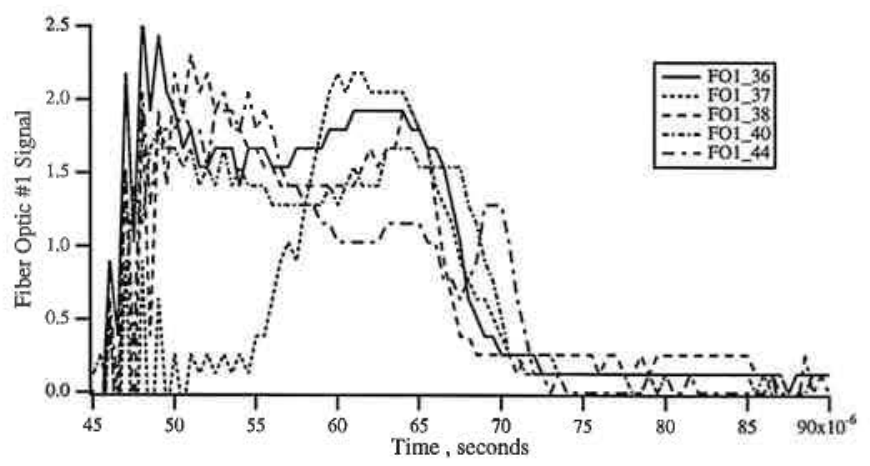

Figure 16. Overlay of fiber optic \#1 waveforms.

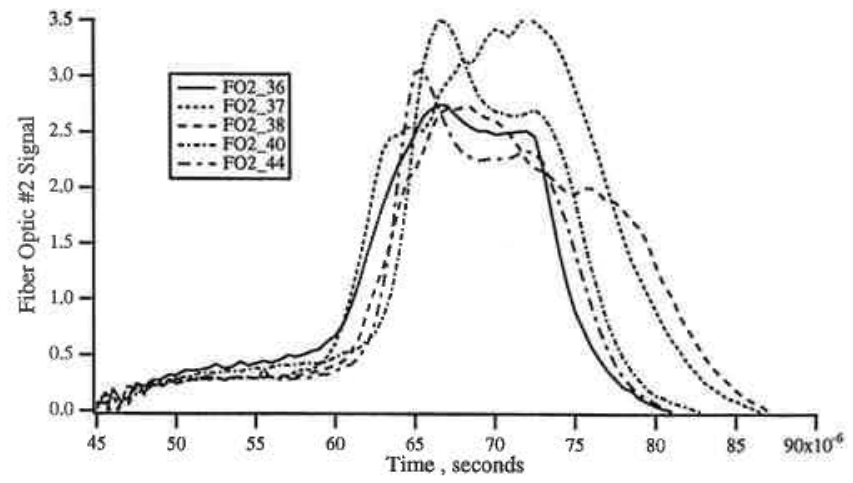

Figure 17. Overlay of fiber optic \#2 waveforms. 


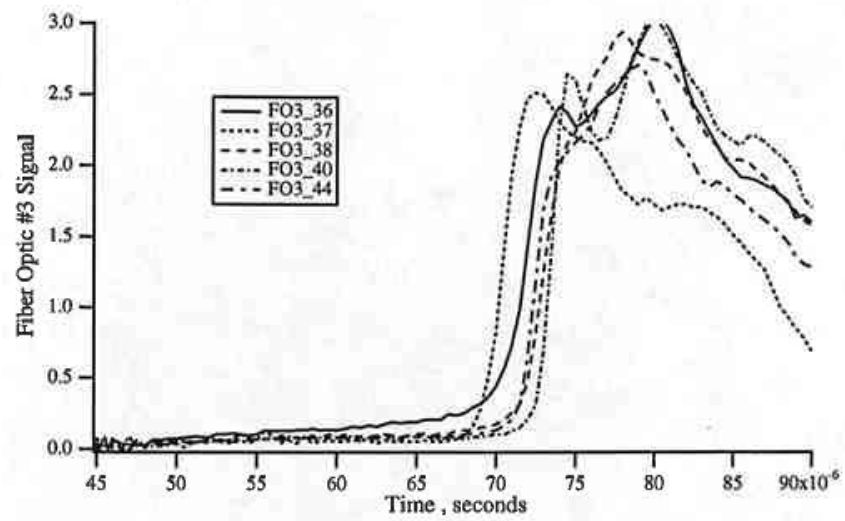

Figure 18. Overlay of fiber optic \#3 waveforms.

A summary of the observations about this data follows:

1) Early time behavior of B-dot probe \#1 shows substantial electrical pick-up from the bank discharge. The shot-to-shot reproducibility is not good, although the late time peak, which probably corresponds to the arc passing this position, is reasonably reproducible in time, but not in amplitude.

2) Except for shots 37 and 38, the signal from B-dot probe \#2 is very reproducible and shows very little timing jitter. The time duration from signal minimum to maximum is about $6 \mu \mathrm{s}$. Combined with a velocity estimate of about 4 $\mathrm{Km} / \mathrm{sec}$ for the arc, this implies a total armature length of 2.4 $\mathrm{cm}$. Based on previous plasma armature experience, this seems to be a reasonable value.

3) Early time response from fiber optic probe \#1 shows UV pickup from discharge initiation onward. This indicates that the probe probably protrudes too far towards the gun bore. A design which ensures better shielding from the arc initiator region should be explored.

4) Fiber optic probes \#2 and \#3 show reproducible leading edge rise and duration, with only about 2 microsecond jitter shot-to-shot. Because they will also see light radiation from the compressed argon gas column being driven by the arc armature, it is not possible to take pulse width times velocity as the total arc length. The signal duration is typically more like $9 \mu \mathrm{s}$, suggesting that the compressed gas length is about $1.2 \mathrm{~cm}$. This is not too much different than simulation code predictions of a gas column length of about $1.1 \mathrm{~cm}$ at this location in the gun (i.e. at $6.5 \mathrm{~cm}$ from the arc initiator). 5) If shots 37 and 38 are excluded from the data, reproducible waveforms are apparent. The results are consistent with snowplow simulations and support the development of a repetitively operated gun when gun materials for the rails and insulators are optimized.

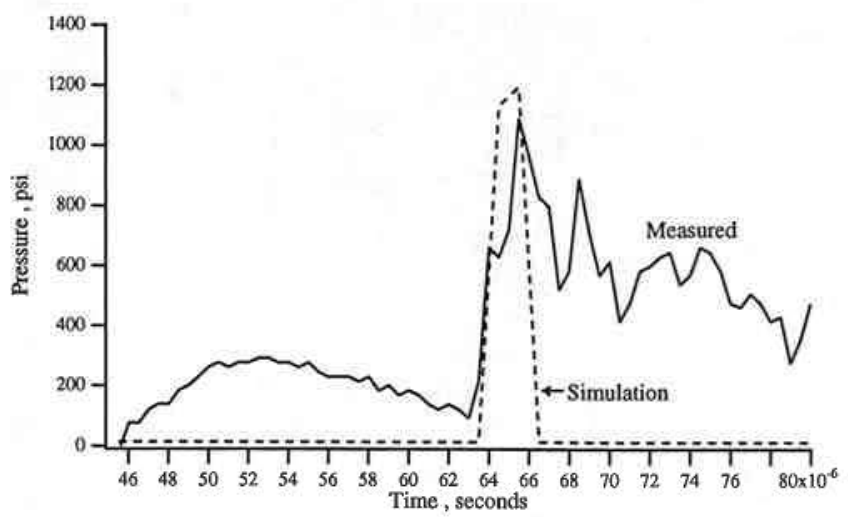

Figure 19. A comparison of predicted pressure versus measured at the lower transducer location.

An important signature of the snowplow process was the amplitude and time duration of the pressure associated with the compressed gas column. Figure 19 shows a measured pressure waveform for the upper transducer location and the pressure predicted by the simulation. The excellent agreement on shock front arrival times and the peak pressure indicate that the snowplow process occurred. The disagreement at later times could be do to the plasma armature which contains structure as well as the dynamic response of the Kapton tape when subjected to a pressure impulse.

The best test of snowplow action is to accelerate spherical particles. A series of shots were carried out using 330 micron diameter glass spheres inserted into the gun bore. The spheres were introduced by placing them on the tip of a fiber optic inserted through the sidewall of the gun. The spheres were at rest at the beginning of the shot, that is they were introduced into the gun bore with zero velocity. The Hadland camera was used to photograph these particles in flight. Figure 20 shows a group of particles in flight.

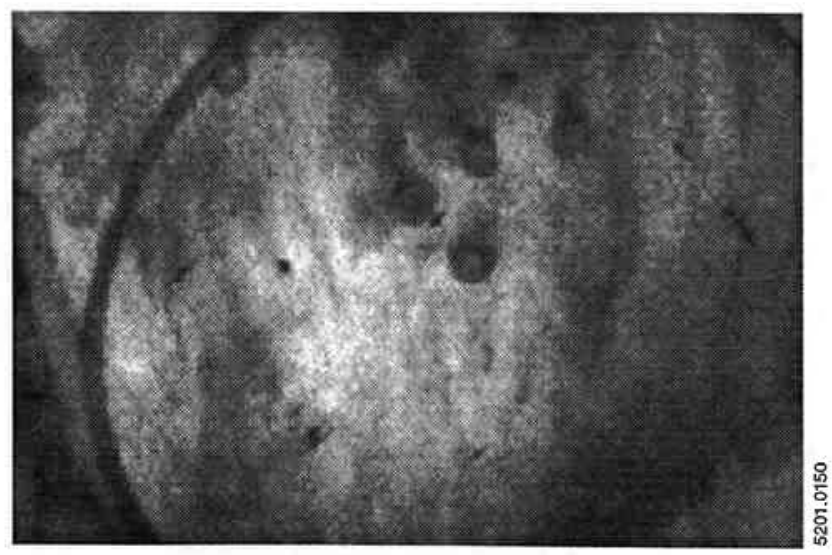

Figure 20. Sixth frame from a sequence of eight used to determine particle velocity. 
Most observed glass sphere velocities were about 600 $\mathrm{m} / \mathrm{sec}$. The simulation code predicted a peak velocity of 625 $\mathrm{m} / \mathrm{sec}$. This was excellent agreement given the problems associated with picking a particular location on a particle from frame to frame when determining velocity.

\section{Conclusion}

A number of different diagnostics were employed to verify the snowplow action of the railgun. All of the data indicates that the railgun is working and matching simulation results. The next railgun will have ceramic sidewalls with no penetrations for pressure transducers, so the primary diagnostic tool will be the B-dot probes. It was necessary to prove that the B-dot probes could be relied upon and the excellent correlation with the data from the other diagnostic tools has verified their accuracy.

\section{Acknowledgment}

This research was sponsored by the United States Air Force, Tinker Air Force Base at Oklahoma City, through a contract administered by ARINC Corporation.

\section{References}

1 J. Uglum, J. Bacon, D. Davis, R. Polizzi, B. Sledge, R. Zowarka, "Scaling Analysis of the Electromagnetic Powder Deposition Gun", Proceedings of this conference.

2 J.W. Shearer, "Xenon Shock Waves Driven by High Magnetic Fields", Proc. 2nd International Conf. On Megagauss Magnetic Fields, 1979.

3 K.A. Jamison, M. Marquez-Reines, and Henry S Burden, " Measurements of the Spatial Distributions of Current in a Rail Gun Arc Armature", IEEE Transactions on Magnetics, Vol. Mag-20, No. 2, March 1984.

4 Jerald V. Parker, "Magnetic-Probe Diagnostics for Railgun Plasma Armatures", IEEE Transactions on Plasma Science, Vol. 17, No. 3, June 1989. 